\title{
A Packet Delivery Cost Analysis of a Flow- Enabled Proxy NEMO Scheme in a Distributed Mobility Anchoring Environment
}

\author{
Shayla Islam ${ }^{1}$, Mohammad Kamrul Hasan ${ }^{2,}$, Aisha Hassan Abdalla Hashim ${ }^{3}$ \\ ${ }^{1}$ Institute of Computer Science and Digital Innovation, UCSI University, \\ 56000 Kuala Lumpur (South Wing), Malaysia \\ ${ }^{2}$ Network and Communication Technology Lab, Faculty of Information Science and Technology, \\ Universiti Kebangsaan Malaysia (UKM), \\ 43600 UKM, Bangi, Malaysia \\ ${ }^{3}$ Department of Electrical and Computer Engineering, International Islamic University Malaysia, \\ 53100 Kuala Lumpur, Malaysia \\ mkhasan@ukm.edu.my
}

\begin{abstract}
DMM (Distributed Mobility Management) is a present elective worldview for creating a mobility management scheme to discourse the centralized issues in present IP-based mobile environments. The main reason is to enable these schemes to adapt to the present increment in the number of mobile operators, as well as mobile information traffic size, just as the pattern in the mobile Internet towards Industry 4.0 in a flat architecture. Until this point, the advancement of schemes dependent on the DMM-based method is still at fundamental phases in the Internet Engineering Task Force (IETF), as well as there is no present standard set up. With the point of taking advantage of utilizing different interfaces all at once, this paper proposes an enhanced Flow-enabled Proxy NEMO scheme in a Distributed Mobility Anchoring (FPNEMO-DMA) environment. Besides, a mathematical approach is advanced to assess the performance of the proposed FPNEMO-DMA scheme and benchmark with the existing Nemo Basic Support Protocol (NBSP) and Proxy NEMO.
\end{abstract}

Index Terms-Distributed mobility anchoring; NBSP; Proxy NEMO; Flow mobility.

\section{INTRODUCTION}

NBSP (Network Mobility Basic Support Protocol) depends on MIPv6 (Mobile IPv6) standards, and it utilizes a centralized mobility management procedure that prompts challenges such as extra signaling overhead, which infers longer handoff latencies, and the vulnerabilities due to the occurrence of a single point of system failure [1]-[8]. With these confinements, the Internet Engineering Task Force (IETF) has lately proposed the Distributed Mobility Management (DMM) worldview that can be considered as a novel and promising way to deal with manage mobility [9][13]. DMM architecture maintains a strategic distance from pointlessly long courses. It is progressively scalable with the expanding number of mobile clients, as well as is an advantageous stage for DMM that means giving mobility support to mobile clients just when they require the support.

Manuscript received 17 January, 2020; accepted 20 May, 2020.
DMM can keep away from misuse of resources, and diminish extra signaling overhead and total network cost.

Notwithstanding, regardless of the way that various mobility management approaches are being structured towards a progressively disseminated activity planning to relieve issues identified with brought together activity, there are occasions where DMM brings about greater expenses, as well as the performances of the system, maybe undermined.

This paper, consequently, proposes an enhanced Flowenabled Proxy NEMO scheme in a Distributed Mobility Anchoring (FPNEMO-DMA) environment. The scheme expands our prior works [14]-[17] that applied the logical interface concept of the LMA (Local Mobility Anchor) in the Proxy NEMO (PNEMO) environment during inter technology handoff to support mobility management.

In this paper, the main contributions are twofold mainly: (i) to propose an enhanced Flow mobility scheme in a Proxy NEMO-based Distributed Mobility Anchoring (FPNEMODMA) environment to alleviate the Packet Delivery Cost (PDC) during inter technology handoff; (ii) to propose a comprehensive performance appraisal through developing a mathematical approach that studies the performance improvements of the proposed FPNEMO-DMA scheme. Then the proposed scheme benchmarked with existing centralized NBSP and Proxy NEMO. Moreover, the proposed scheme investigates and analyzes the Packet Delivery Cost (PDC) in terms of a varying number of mobile routers, cell residence time, and average session length.

\section{RELATED WORK}

The NBSP characterizes a mobile network as a system fragment that can travel, as well as append, to a subjective point in the routing substructure [6]-[8]. It is made from at any rate one MR (Mobile Router) and different Mobile Network Nodes (MNNs). The MR gives access to the MNNs to associating with the outside foundation, and it is responsible for managing the mobility of MNNs inside the 
mobile environment.

The NBSP entails a HA (Home Agent) like the individual in MIPv6; the HA enlists the mobile network's area, as well as advances packets to it. To accomplish these roles, the HA does three essential consistent functions that are mainly: (i) the allotment of home network address for an MR, (ii) managing the location, which includes overseeing and monitoring the internetwork area for the MR (this includes the mapping of the Home Address (HoA) to a location where the MR is accessible), and (iii) managing routing, catching parcels from the HoA of the MNN, as well as sending the bundles in the view of the internetwork area data from the Location Management (LM) - either to the goal or to some other system component that realizes how to advance them to the goal. Figure 1 delineates the NBSP architecture.

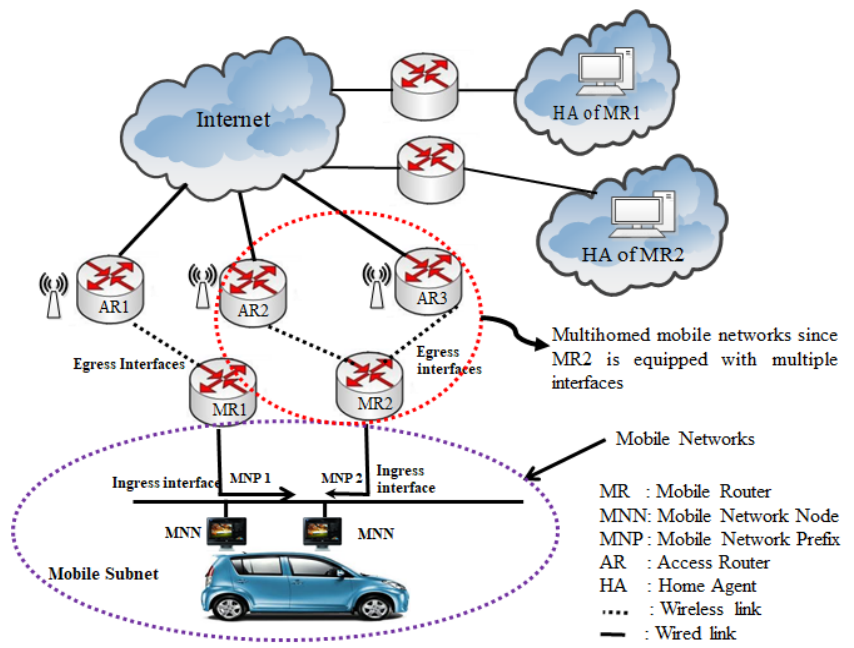

Fig. 1. The basic architecture of NBSP.

Proxy NEMO is named as a network-based mobility scheme that does not require any software on the MR during movement among diverse access networks [6]-[8]. Proxy NEMO presents two system substances, to be specific - the LMA and the Mobility Access Gateway (MAG). The previous is the HA for the MR in a PMIPv6 area, where the mobility is overseen by Proxy NEMO. The last is an access gateway that deals with the mobility-related to signaling for an MR, which is appended to it. The architecture of Proxy NEMO is presented in Fig. 2.

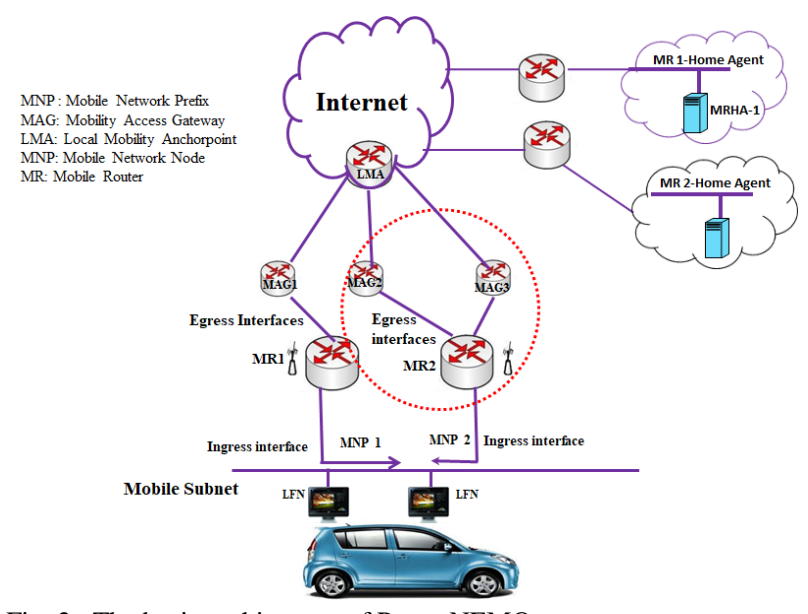

Fig. 2. The basic architecture of Proxy NEMO.
Contrasted with the NBSP, Proxy NEMO can lessen the handoff delay as far as mobility discovery and copied address identification; nevertheless, it has various confinements. For instance, in situations where all information parcels and signaling messages are directed to the LMA, bringing about a potential single point of failure To solve these constraints, the architecture of DMM splits the functionality of LMA to numerous MAGs that are set at the edge of the system. From the practical dispersion, the DMM can accomplish improving scalability issues, avoiding a single point of failure reducing signaling overhead cost and PDC, etc. [11]-[15].

A DMM-based framework utilizes a network-based mobility concept and integrates the functionality of LMA, as well as MAG of ProxyMIPv6, in each access gateway. A Proxy NEMO-based DMM framework for the mobile network is developed for nested, as well as non-nested, scenarios to reduce the packet delivery cost in NEMO [11]. The schemes break down the LMA element in PMIPv6 and disperse the functionality of routing management to the entries of the altered networks as represented in Fig. 3.

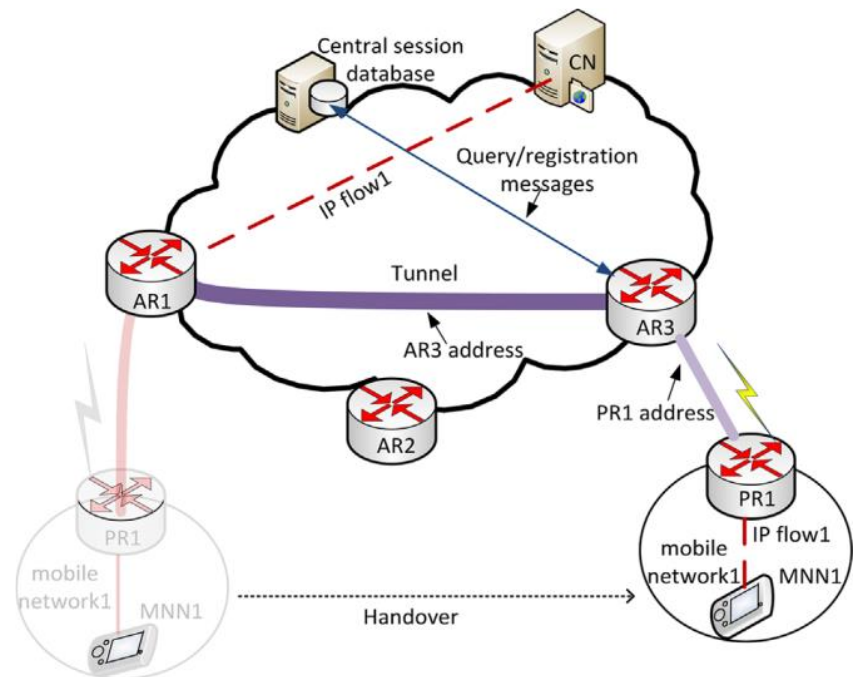

Fig. 3. Basic architecture of DMM-based NEMO framework [11].

\section{PROPOSED FPNEMO-DMA ARCHITECTURE}

A significant prerequisite in DMM is to allow traffic flow to abstain from navigating a single mobility anchor a long way from the ideal route. In this section is proposed an enhanced Flow mobility scheme in a Proxy NEMO-based Distributed Mobility Anchoring (FPNEMO-DMA) environment is proposed to alleviate the extra signaling overhead cost at the time of inter technology handoff.

As indicated by the proposed framework, the Flowenabled Control-Plane Anchor (FCPA), just as the SMR (serving MR), acts like an LMA, as well as MR in PNEMO environment. The Flow-enabled Distributed Data Plane Anchors (FDDPAs) are utilized as substitution of MAG in PNEMO. The FDDPAs are subject to distinguishing the serving MR's movements amongst diverse access networks. They are additionally capable to separate, as well as achieve the Mobile Network Prefix (MNP) just as Home Network Prefix (HNP) from an affirmation message that is coordinated from the nearby HA. Figure 4 shows a general scenario of the proposed FPNEMO-DMA scheme. 


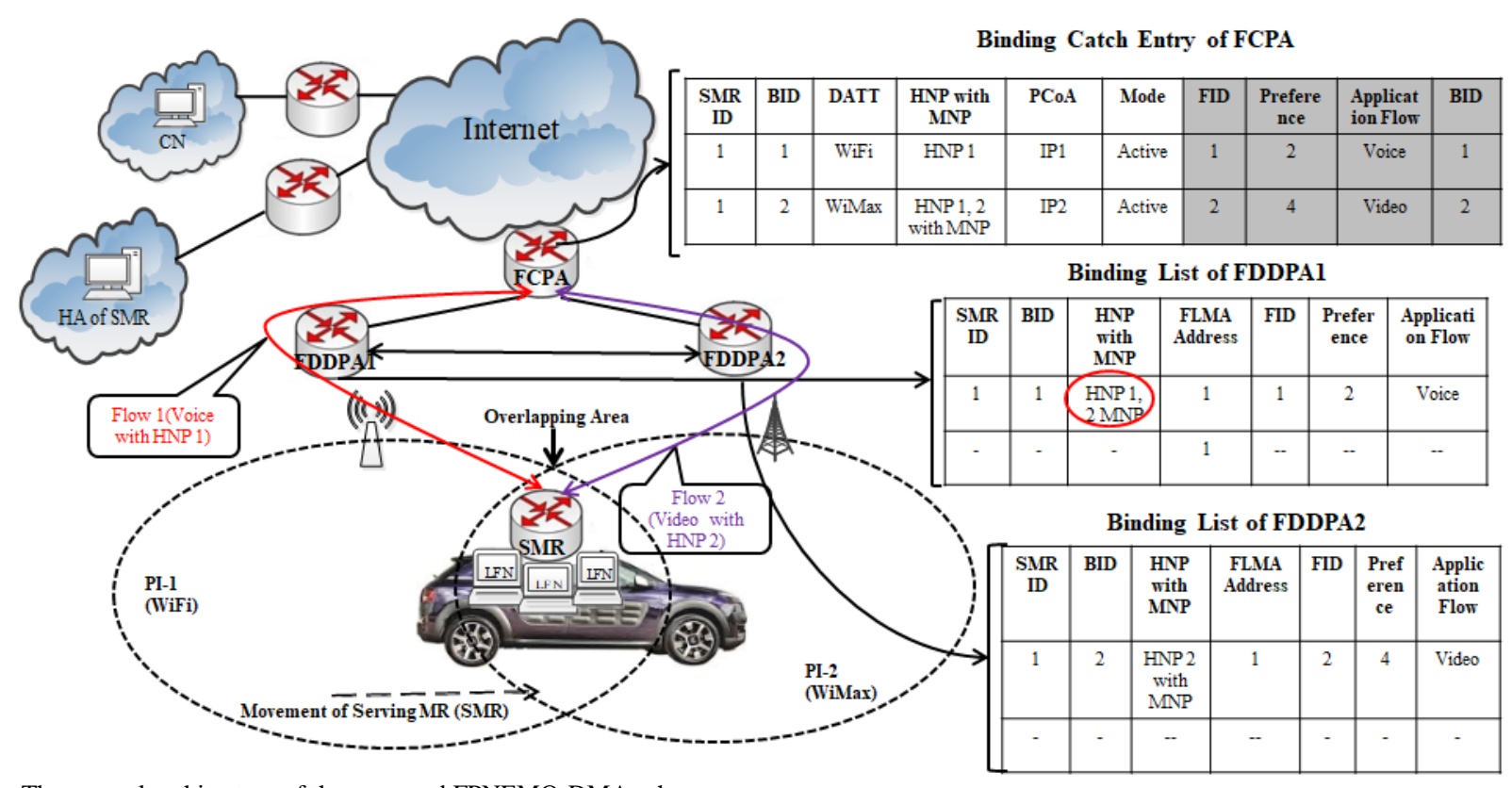

Fig. 4. The general architecture of the proposed FPNEMO-DMA scheme.

In the FPNEMO-DMA scheme, the serving MR picks as the source address for data packets having a place with an IP flow, a location designated by the network where the serving MR is appended to when the flow is started. At the point when a serving MR appends to a network (physical interface 1) or moves to another network (physical interface 2), an IP prefix from the joined network is allotted to the serving MR's interface. Notwithstanding designing new connection local areas, serving MR arranges an IP address from this prefix that is commonly a dynamic IP address. It at that point utilizes this IP address once a flow is started. Packets from this flow routed to the serving MR are just sent by the progressing table.

There might be various IP discourses that a serving MR can choose while starting a flow. They might be from a similar or distinctive access network. The network may promote these prefixes with cost choices, so the serving MR can pick the one with the minimum cost. Moreover, these IP prefixes might be of various sorts concerning whether mobility support is required. A serving MR should pick which IP prefix to use for each flow as indicated by whether it needs IP mobility support or not. At the point when the serving MR travels, the past anchor still anchors the traffic utilizing the old IP address and advances it to the new serving MR's area. The serving MR acquires another IP address secured to the new area, as well as ideally utilizes it for new correspondences, set up while associated with the new area. The mobility anchor remains to assume that job and advances traffic to another locator in the new network as illustrated in Fig. 5.
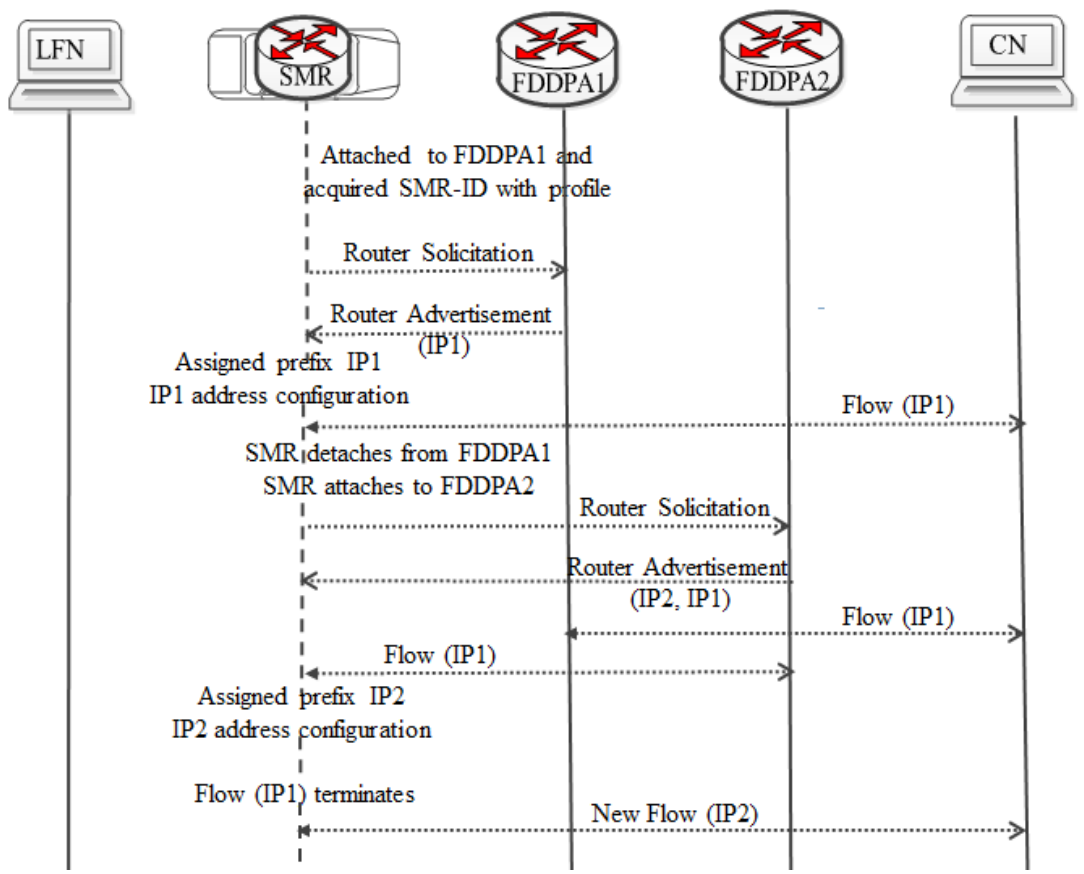

Fig. 5. Signaling Flow during the movement of Serving MR to a new network. 


\section{A. Message Format}

A new Flow-based Mobile Network Anchored Prefix (FMNAP) preference is stated for usage with the Proxy Binding Update (PBU) as well as Proxy Binding Acknowledgment (PBA) messages traded among FCPA and FDDPAs (shown in Fig. 6). Accordingly, this alternative can show up if the D bit is fixed in a PBU or PBA message. This alternative is utilized for trading the serving MR's prefix attached to the network-based domain.

\section{B. The Distributed Logical Interface (DLIF)}

Distributed Logical Interface (DLIF) (see Fig. 7) is a logical interface that permits to effortlessly conceal the difference in related anchors from the serving MR. Along these lines, it is conceivable to enable a serving MR to at the same time send or get traffic, which is tied down at various mobility anchors, as well as an access router, and to effect on the serving MR selection procedure of its home IPv6 address for another flow, except the need of exceptional support from the serving MR IP stack.
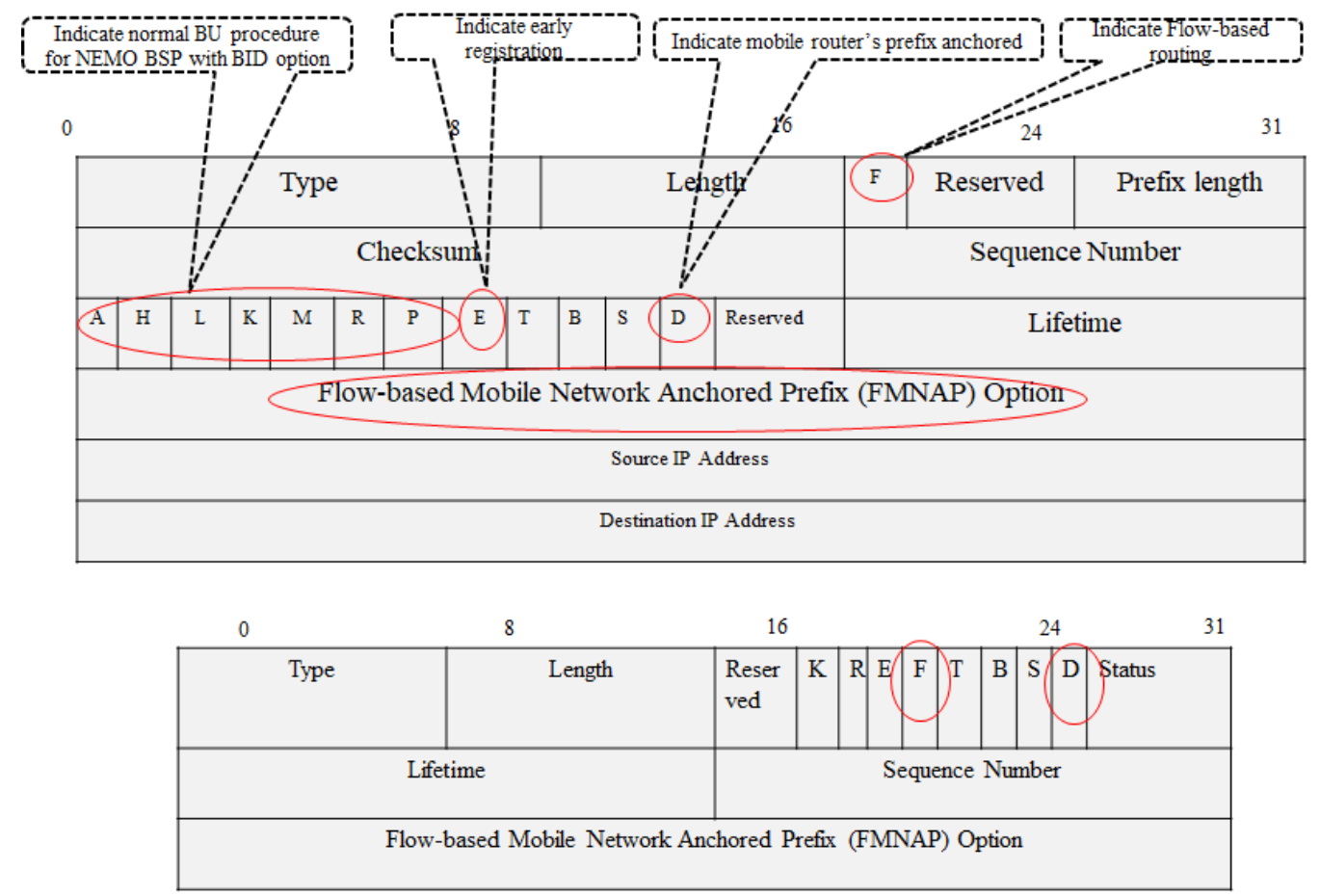

Fig. 6. Extended PBU and PBA message formats.

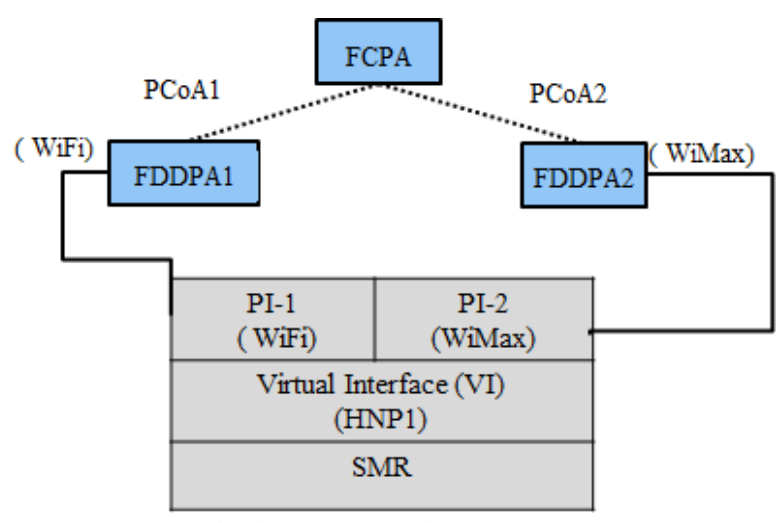

Fig. 7. DLIF structure in the proposed scheme.

\section{PACKet Delivery Cost (PDC) ANALYSIS}

Assessment of the proposed FPNEMO-DMA scheme is concentrated on calculations utilizing a mathematical model as depicted in Fig. 8. The performance of the FPNEMODMA scheme is analyzed, as well as benchmarked with NBSP [5] and Proxy NEMO [7]. Proxy NEMO is contrasted with FPNEMO-DMA scheme, because the two schemes use network-based mobility concepts to address NEMO issues, particularly for the analysis of PDC.

The PDC includes both the packet transmission and preparing costs. Thusly, the absolute PDC can be termed as the straight relationship of packet Tunneling Cost (TC) and Loss Cost (LC) [5], [7].

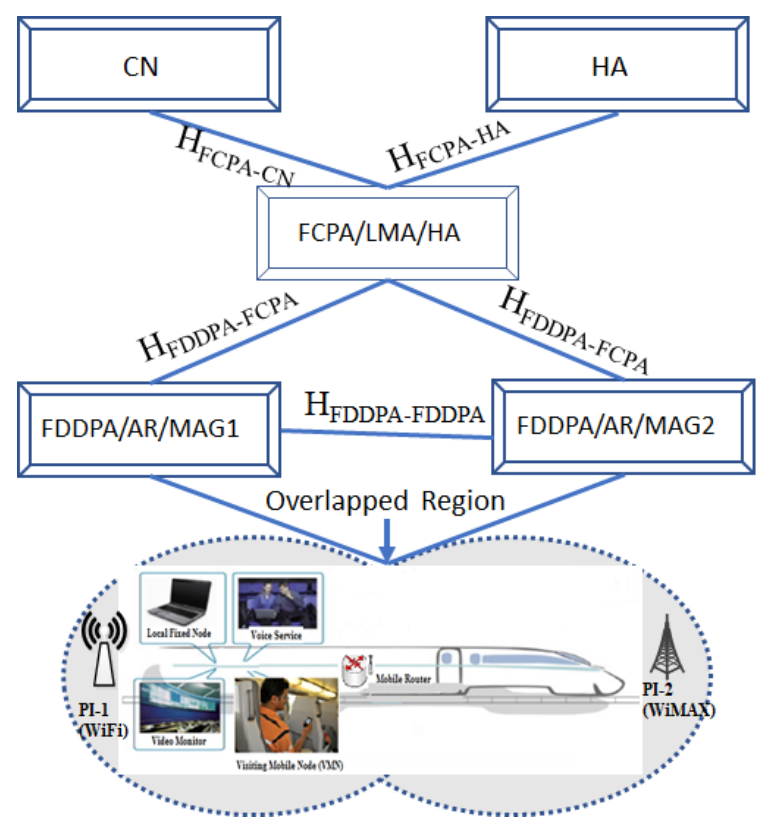

Fig. 8. Mathematical model of the proposed FPNEMO-DMA scheme.

FPNEMO-DMA scheme incorporates logical interfacebased flow mobility in PNEMO environment. Henceforth, 
the FCPA of the serving MR upholds the BCE as HA of MR in NBSP. Accordingly, PDC of FPNEMO-DMA scheme can be calculated as the total of the TC. In this way, the PDC of FPNEMO-DMA, NBSP, and Proxy NEMO can be expressed as

$$
\partial_{P D C}^{F P N E M O-D M A}=\left[\begin{array}{l}
N_{C N} \times N_{M R} \times A S L \times \mu_{h} \times \\
\times\left\{\left(\begin{array}{l}
\theta_{\text {success }} \times \tau \times \\
\times C P N E M O-D M A
\end{array}\right)\right\}
\end{array}\right],
$$

where $C_{T C}^{F P N E M O-D M A}$ can be defined as

$$
\left.C_{T C}^{F P N E M O-D M A}=\left\{\begin{array}{l}
\mathrm{H}_{F D D P A-F D D P A} \times\left(2 \times L_{T H D}\right) \times \\
\max \left(\begin{array}{l}
H_{F C P A-F D D P A} \times \\
\times t_{w d} \\
-\mathrm{H}_{F D D P A}-F D D P A \\
\times t_{w d}, 0
\end{array}\right), \\
\left(\begin{array}{l}
T_{L S}+2 \times \\
\times H_{F C P A}-F D D P A \\
\times t_{w d}
\end{array}\right)
\end{array}\right)\right\}
$$

As indicated by the NBSP handoff procedure, encapsulated packets are passed through the passage in the middle of the MR, as well as it's HA. This prompt improve tunneling cost (TC). Moreover, packets bound for Local Fix Nodes (LFNs) are passed on from the Corresponding Node (CN) employing the access gateway over the wireless connection during handoff. Therefore, packet loss cost is included with the tunneling cost. This is because data packets are received at the destination interface because of the loss of remote availability among the access gateway, as well as the MR. Along these lines, the PDC of the NBSP is expressed as

$$
\Psi_{P D C}^{N B S P}=N_{M R} \times A S L \times \mu_{h} \times\left(\tau \times C_{T C}^{N B S P}+\sigma \times C_{\text {Loss }}^{N B S P}\right),
$$

where:

$$
\begin{aligned}
& C_{T C}^{N B S P}=\left\{\begin{array}{l}
\left(\frac{P_{w l f} \times \mathrm{H}_{A R}-M R}{1-P_{w l f}} \times L_{T H D}\right)+ \\
+\left(\mathrm{H}_{H A}-A R \times L_{T H D}\right)
\end{array}\right), \\
& C_{P L}^{N B S P}=\left(\mathrm{H}_{C N}-H A+\mathrm{H}_{H A-A R} \times L_{T H D}\right) \times \\
& \times\left(T_{H D}^{N B S P}-\frac{T_{R E G}}{2}\right) .
\end{aligned}
$$

Contrasted with NBSP, the data tunnel is made in the middle of the LMA and the MAG2 in the Proxy NEMO scheme. Accordingly, tunneled data packets are not delivered on over the remote connection. Along these lines, the PDC of the Proxy NEMO is expressed as:

$$
\begin{aligned}
& \Psi_{P D C}^{\operatorname{Pr} \text { oxyNEMO }}=N_{M R} \times A S L \times \mu_{h} \times \\
& \times\left(\tau \times C_{T C}^{\operatorname{Pr} \text { oxyNEMO }}+\sigma \times C_{P L}^{\operatorname{Pr} \text { oxy } N E M O}\right),
\end{aligned}
$$

where

$$
C_{T C}^{\operatorname{Pr} \text { oxyNEMO }}=\left(\mathrm{H}_{L M A}-M A G \times L_{T H D}\right) .
$$

\section{RESULTS AND DISCUSSION}

This section talks about the performance assessment of FPNEMO-DMA, NBSP, and Proxy NEMO. The default estimations of the system parameters are thought to be as per the following [14], [16]: number of serving $M R=20$, link switching delay $=300 \mathrm{~ms}$, failure probability for wireless link $=0.5$, bandwidth of wireless link $=54 \mathrm{Mb} / \mathrm{s}$, wired link bandwidth $=1000 \mathrm{Mb} / \mathrm{s}$, length of RA $=92$ bytes, and cell residence time $\left(\mathrm{T}_{\mathrm{CR}}\right)=10$ seconds.

The comparative PDC depending on tunneling weight factor $(\tau)$, Average Session Length (ASL), and the number of serving MR are plotted in Figs. 9-12. Usually, $\tau$ increases as soon as the traffic density on that access link accelerates.

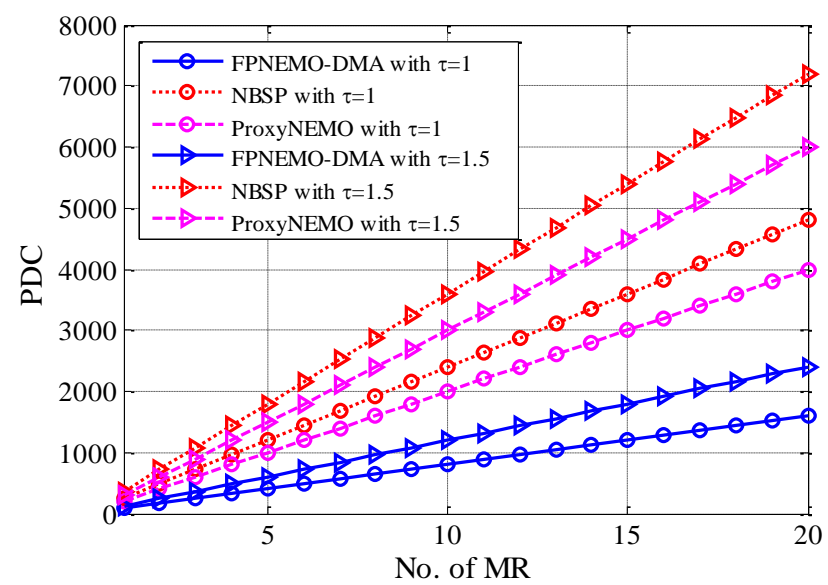

Fig. 9. PDC vs. No. of serving MR $(\mathrm{ASL}=10)$.

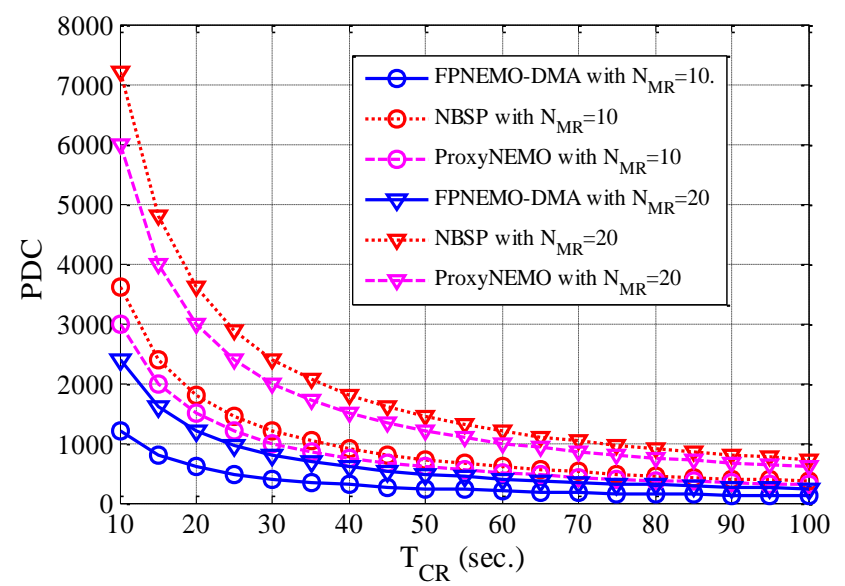

Fig. 10. PDC vs. $\mathrm{T}_{\mathrm{CR}}$.

Figure 9 and Figure 10 illustrate the impact of the quantity of the serving MR, as well as residence time on PDC. It is noticed from both figures, that while ASL is set as 10, the number of serving MR, as well as TCR, is changing. As the proposed framework integrates the DMM-based flow 
mobility concept on a proxy domain, it is delicate to the measure of sessions that are effectively running equal and the Flow enable Controlled Plane Anchor (FCPA) space size too. If the number of serving MR increases with lower TCR, the packet handling and directing cost increases at FLMA. Consequently, it brings about expanded PDC. In any case, it is seen from the two figures that the PDC of NBSP is greater compared to FPNEMO-DMA and Proxy NEMO. This is on the grounds, that if there should arise an occurrence of NBSP, numerous tunneled packets should be directed to the serving MR from $\mathrm{CN}$ utilizing $\mathrm{HA}$ of the serving MR for every movement.

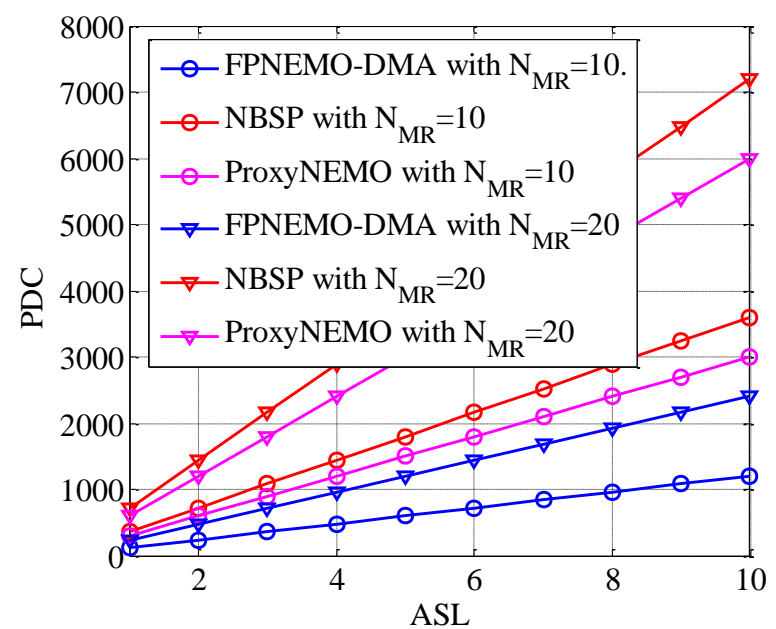

Fig. 11. PDC vs. ASL (packets/flow).

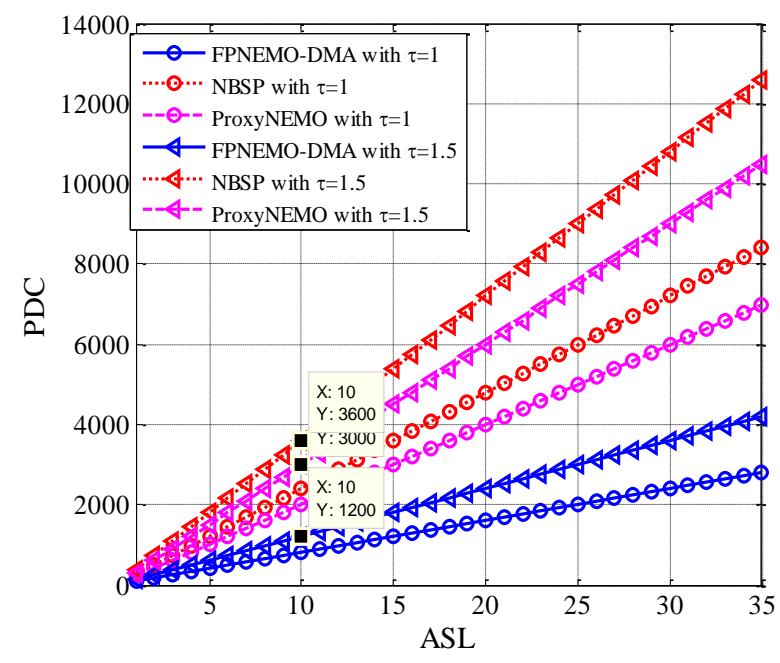

Fig. 12. PDC vs. TCR.

As presented in Fig. 11 and Fig. 12, the PDC has expanded relatively with the ASL for all schemes (i.e., FPNEMO-DMA, Proxy NEMO, and NBSP). In any case, the tunneling costs of Proxy NEMO increments as $\tau$ increments. As a consequence, tunneling burdens increments with expanding the quantity of serving MRs. This outcome in extreme congestion. The proposed FPNEMO-DMA expends lower PDC compared to NBSP, as well as Proxy NEMO, as the ASL and $\tau$ increments. Precisely, when $\tau$ is high, the FPNEMO-DMA extensively bypasses the Proxy NEMO. The explanation is that the DMM-based flow mobility concept is integrated on the Proxy domain and the route among the FDDPAs is not utilized in the FPNEMO-
DMA architecture. Moreover, all flows are directed recurrently alongside with the preference settings in the proposed scheme. Therefore, FPNEMO-DMA exterminates the burden on the tunnel among various access routers and lessens the impact of any possible shutdown of the tunnel. Thus, the proposed scheme essentially lessens the tunnelling cost. Subsequently, it is more appropriate for real-time application scenarios.

\section{CONCLUSIONS}

In this paper, an enhanced Flow-enabled Proxy NEMObased scheme in a Distributed Mobility Anchoring (FPNEMO-DMA) environment is proposed with the point of taking advantage of utilizing different interfaces at the same time. The considered performance metric to assess the performance of the FPNEMO-DMA is PDC. According to mathematical analysis, the PDC for the proposed FPNEMODMA scheme has been observed to be lower compared to the NBSP and proxy NEMO. This is because of the influence of the changed number of the serving MR on PDC is found to be much higher in NBSP compared to the proposed FPNEMO-DMA and Proxy NEMO schemes.

\section{APPENDIX A}

The notations and symbols used in this paper are listed in Table I.

TABLE A-I. THE ACRONYM LIST.

\begin{tabular}{|c|c|}
\hline Notation & Description \\
\hline ASL & Average Session Length \\
\hline FCPA & Flow-enabled Control Plane Anchor \\
\hline FDDPAs & Flow-enabled Distributed Data Plane Anchors \\
\hline HI & Home Network Prefix \\
\hline HNP & Hop distance from X-Y \\
\hline HX-Y & Longth \\
\hline L & Multiple Care of Address \\
\hline LMA & Number of Correspondent Node \\
\hline MCoA & Number of Mobile Router \\
\hline NCN & Network Mobility \\
\hline NMR & Proxy Mobile IPv6 \\
\hline NEMO & Link Switching Delay \\
\hline PMIPv6 & \\
\hline TLS &
\end{tabular}

\section{CONFLICTS OF INTEREST}

The authors declare that they have no conflicts of interest.

\section{REFERENCES}

[1] I. Al-Surmi, M. Othman, and B. M. Ali, "Mobility management for IP-based next generation mobile networks: Review, challenge and perspective", Journal of Network and Computer Applications, vol. 35, no. 1, pp. 295-315, 2012. DOI: 10.1016/j.jnca.2011.09.001.

[2] B. Chandavarkar and G. Ram Mohad Reddy, "Survey paper: Mobility management in heterogeneous wireless networks", Procedia Engineering, vol. 30, pp. 113-123, 2012. DOI: 10.1016/j.proeng.2012.01.841.

[3] J. P. Jeong, S. Cespedes, N. Benamar, and J. Haerri, "Survey on IPbased vehicular networking for intelligent transportation systems", Internet Engineering Task Force (IETF), 2016. [Online]. Available: Internet-Draft draft-jeong-its-vehicular-networking-survey https://datatracker.ietf.org/doc/draft-jeong-ipwave-vehicularnetworking-survey/

[4] R. Wakikawa, V. Devarapalli, G. Tsirtsis, T. Ernst, and K. Nagami, 
"Multiple care-of addresses registration", RFC 5648, 2009. DOI: $10.17487 /$ rfc5648

[5] Ch.-W. Lee, M. Ch. Chen, and Y. S. Sun, "A novel network mobility management scheme supporting seamless handover for high-speed trains", Computer Communications, vol. 37, pp. 53-63, 2014. DOI: 10.1016/j.comcom.2013.09.009.

[6] J.-H. Lee, T. Ernst, and N. Chilamkurti, "Performance analysis of PMIPv6-based network mobility for intelligent transportation systems", IEEE Transactions on Vehicular Technology, vol. 61, no. 1, pp. 74-85, 2012. DOI: 10.1109/TVT.2011.2157949.

[7] S. Ryu, J.-W. Choi, and K.-J. Park, "Performance evaluation of improved fast PMIPv6-based network mobility for intelligent transportation systems", Journal of Communications and Networks, vol. 15, no. 2, pp. 142-152, 2013. DOI: 10.1109/JCN.2013.000027.

[8] J.-H. Lee, and T. Ernst, "Lightweight network mobility within PMIPv6 for transportation systems", IEEE Systems Journal, vol. 5 , no. 3, pp. 352-361, 2011. DOI: 10.1109/JSYST.2011.2158681.

[9] J. Carmona-Murillo, I. Soto, F. J. Rodríguez-Pérez, D. Cortés-Polo, and J. L. González-Sánchez, "Performance evaluation of distributed mobility management protocols: Limitations and solutions for future mobile networks", Mobile Information Systems, vol. 2017, 15 pages, 2017. DOI: $10.1155 / 2017 / 2568983$.

[10] C. J. Bernardos et al., "Proxy mobile IPv6 extensions for distributed mobility management", 2019. [Online]. Available: https://tools.ietf.org/html/draft-ietf-dmm-pmipv6-dlif-04

[11] P. P. Ernest, O. E. Falowo, and H. A. Chan, "Design and performance evaluation of distributed mobility management schemes for network mobility", Journal of Network and Computer Applications, vol. 61, pp. 46-58, 2016. DOI: 10.1016/j.jnca.2015.09.012

[12] H. Chan, S. Jeon, J. Lee, X. Wei, and F. Templin, "Distributed mobility anchoring", 2015. [Online]. Available: https://tools.ietf.org/html/draft-chan-dmm-distributed-mobilityanchoring-06

[13] A. Leiter, L. Bokor, "A flow-based and operator-centric dynamic mobility management scheme for proxy mobile IPv6", Wireless Communications and Mobile Computing, vol. 2019, 21 pages, 2019. DOI: $10.1155 / 2019 / 4567317$.

[14] S. Islam, A.-H. A. Hashim, M. H. Habaebi, and M. K. Hasan, "Design and implementation of a multihoming-based scheme to support mobility management in NEMO", Wireless Personal Communications: An International Journal, vol. 95, no. 2, pp. 457473, 2017. DOI: 10.1007/s11277-016-3903-7.

[15] R. Kuntz, J. Montavont, and T. Noel, "Multihoming in IPv6 mobile networks: Progress, challenges, and solutions", IEEE Communications Magazine, vol. 51, no. 1, pp. 128-135, 2013. DOI: 10.1109/MCOM.2013.6400449.

[16] Sh. Islam, A. H. Abdalla, and M. K. Hasan, "Novel multihomingbased flow mobility scheme for proxy NEMO environment: A numerical approach to analyse handoff performance", SCIENCEASIA, vol. 43, pp. 27-34, 2017. DOI: 10.2306/scienceasia1513-1874.2017.43S.027.

[17] Sh. Islam, A. H. A. Hashim, and M. K. Hasan, "A resource-efficient mobility management scheme to analyze handoff performance in PNEMO environment", in Proc. of 2019 7th International Conference on Mechatronics Engineering (ICOM), 2019. DOI: 10.1109/ICOM47790.2019.8952058.

This article is an open access article distributed under the terms and conditions of the Creative Commons Attribution 4.0 (CC BY 4.0) license (http://creativecommons.org/licenses/by/4.0/). 